\title{
Neodymium-doping Concentration Induced Face- shared to Corner-shared Transition in Strontium Cobaltite
}

Haoliang Huang ( $\sim$ hhliang@ustc.edu.cn )

Anhui Laboratory of Advanced Photon Science and Technology, Hefei National Laboratory for Physical

Sciences at the Microscale, University of Science and Technology of China, Hefei 230026, P. R. China https://orcid.org/0000-0002-5686-5519

\section{Zezhi Chen}

University of Science and Technology of China

Liang Xie

North China University of Technology

Jianlin Wang

University of Science and Technology of China

\section{Ranran Peng}

University of Science and Technology of China

\section{Zhengping Fu}

University of Science and Technology of China

\section{Yalin Lu}

University of Science and Technology of China

\section{Research Article}

Keywords: ceramics, solid state reactions, crystal structure, magnetic measurements, X-ray diffraction, Xray absorption spectroscopies

Posted Date: October 21st, 2020

DOI: https://doi.org/10.21203/rs.3.rs-94018/v1

License: (1) (i) This work is licensed under a Creative Commons Attribution 4.0 International License.

Read Full License

Version of Record: A version of this preprint was published at Journal of Materials Science: Materials in Electronics on March 9th, 2021. See the published version at https://doi.org/10.1007/s10854-021-05593-

3. 



\section{Neodymium-doping Concentration Induced Face- shared to Corner-shared Transition in Strontium Cobaltite}

Haoliang Huang, ${ }^{\mathrm{a}, *}$ Zezhi Chen, ${ }^{\mathrm{b}}$ Liang Xie, ${ }^{\mathrm{c}, *}$ Jianlin Wang, ${ }^{\mathrm{a}}$ Ranran Peng, ${ }^{\mathrm{a}, \mathrm{b}}$ Zhengping $\mathrm{Fu}^{\text {a. }}{ }^{\mathrm{b}}$ Yalin $\mathrm{Lu}^{\mathrm{a}}{ }^{\mathrm{b}, *}$

${ }^{a}$ Anhui Laboratory of Advanced Photon Science and Technology, Hefei National Laboratory for Physical Sciences at the Microscale, University of Science and Technology of China, Hefei 230026, P. R. China

${ }^{b}$ CAS Key Laboratory of Materials for Energy Conversion, University of Science and Technology of China, Hefei 230026, P. R. China

${ }^{c}$ Department of Physics, North China University of Technology, Beijing 100144, P. R. China

E-mail addresses of all authors:

Haoliang Huang: hhliang@ustc.edu.cn, +86 551-63603194

Zezhi Chen: chenzz@mail.ustc.edu.cn

Liang Xie: xieliang@ncut.edu.cn, +86 010-88803271

Jianlin Wang: wangjl@ustc.edu.cn

Ranran Peng: pengrr@ustc.edu.cn

Zhengping Fu: fuzp@ustc.edu.cn

Yalin Lu: yllu@ustc.edu.cn, +86 551-63603004 


\begin{abstract}
The topotactic connection style of oxygen octahedron/tetrahedron in transition metal oxides (TMOs) is an important feature that modulates their corresponding physical properties. Using a simple chemical doping technique, we obtained $\operatorname{Sr}_{1-x} \mathrm{Nd}_{x} \mathrm{CoO}_{3-\delta}$ with a crystal structure transition from face-shared octahedron to corner-shared octahedron/tetrahedron. The Rietveld analyses of the x-ray diffraction (XRD) patterns show that the crystal structure changes from rhombohedral to cubic and the connection style transforms from face-shared to corner-shared with the increase neodymium (Nd) content. During this process, the ferromagnetic behavior is greatly improved due to the larger amount of the corner-shared cubic $\mathrm{SrCoO}_{3-\delta}$ phase. The synchrotron radiation $\mathrm{x}-$ ray absorption spectroscopies of the Co $L$-edge and $\mathrm{O} K$-edge show that $\mathrm{Nd}$ doping mainly affects the electronic structure of oxygen rather than the valence state of Co. Thereby, the Nd changes the connection style of oxygen octahedron/tetrahedron, which then alters the magnetic interactions.
\end{abstract}

Keywords: ceramics; solid state reactions; crystal structure; magnetic measurements; X-ray diffraction; X-ray absorption spectroscopies. 


\section{Introduction}

Transition metal oxides (TMOs) are a material class with abundant and novel physical properties, such as superconductivity, [1] multiferroicity, [2,3] magnetoresistance, [4] and others. Within TMOs, transition metal ions are in the form of tetrahedrons $\left(\mathrm{BO}_{4}\right)$ or octahedrons $\left(\mathrm{BO}_{6}\right)$ due to the different valence states of the cations and/or the concentration of oxygen vacancies. At the same time, the topotactic connection style, such as corner-shared, edge-shared or face-shared, of $\mathrm{BO}_{6}$ or $\mathrm{BO}_{4}$ directly affects the angle and distance of the $B-O-B$ connection. As a result, the corresponding physical properties and the oxygen coordination numbers can be modulated by changing the connection style. For example, Yamamoto et al. synthesized oxyhydride $\mathrm{BaVO}_{3-x} \mathrm{H}_{x}(0.3 \leq x \leq 0.8)$ with a hexagonal $6 \mathrm{H}$ structure having both faceand corner-shared octahedron. [5] This high pressure synthesized material is a quasitwo-dimensional metal with an anomalous temperature dependence of the electrical resistivity and strong antiferromagnetic fluctuations. It can be considered that transition metal oxyhydrides with face-shared or edge-shared octahedral that enable much smaller $B-O-B$ angles resulting to versatile magnetic interactions and thus exhibiting interesting properties. Analogously, Søndenå et al. suggested that a transformation from cornershared to face-shared octahedron can be induced by increasing the alkaline-earth cation concentration. [6] They determined the lowest-energy structure for a series of perovskites $\left(\mathrm{AMnO}_{3}\right.$ where $\left.A=\mathrm{Ca}, \mathrm{Sr}, \mathrm{Ba}\right)$ at low temperatures using density-functional calculations, and pointed out that the charge of $\mathrm{Mn}$ is much lower than that of the traditional ionic model charge due to the covalence of $\mathrm{Mn}-\mathrm{O}$, which reduces the repulsion of Mn-Mn and facilitates the sharing of octahedral faces. Unfortunately, the crystal structure of topotactic connection style is not easily adjusted due to the phenomenon of growth competition. [7] Previous works have reported high pressure oxygen annealing, [8] electrochemical oxidation, [9] and high pressure ( GPa) treatment $[5,10]$ methods to adjust the topotactic connection style. Therefore, it is important to develop a simple and effective method to realize the transformation and understand its effect on the material physical properties. 
Strontium cobaltite, as a type of multivalent oxide, exhibits a transformation from face-shared $\mathrm{Sr}_{2} \mathrm{Co}_{2} \mathrm{O}_{5}$ [9] to corner-shared $\mathrm{SrCoO}_{3}[9]$ or $\mathrm{SrCoO}_{2.5}[11]$ based on the concentration and distribution of oxygen vacancies, as shown in Fig. 1. The cubic $\mathrm{SrCoO}_{3}(\mathrm{C}-\mathrm{SCO})$ consists of corner-shared $\mathrm{CoO}_{6}$ octahedrons and is ferromagnetic (FM), while the rhombohedral $\mathrm{Sr}_{2} \mathrm{Co}_{2} \mathrm{O}_{5}$ (R-SCO) consists of face-shared $\mathrm{CoO}_{6}$ octahedrons and the tetragonal $\mathrm{SrCoO}_{2.5}$ (T-SCO) consists of corner-shared $\mathrm{CoO}_{6}$ and $\mathrm{CoO}_{4}$, which are both antiferromagnetic (AFM). Due to the metastability of $\mathrm{Co}^{4+}$, the $\mathrm{C}$-SCO is not completely close-packed and that $\mathrm{C}-\mathrm{SCO}$ on its own is prone to have oxygen vacancies. [12-14] As a result, the reversible topotactic phase transition between C-SCO and T-SCO has sparked interest due to the enhanced catalytic activities by oxygen sponges for sensors and solid oxide fuel cells. $[15,16]$ However, controlling the concentration and distribution of oxygen vacancies in an SCO system requires unique annealing conditions $[16,17]$ or chemical oxidation. [18] What's more, the faceshared R-SCO is rare and difficult to convert between the above phases. [9]

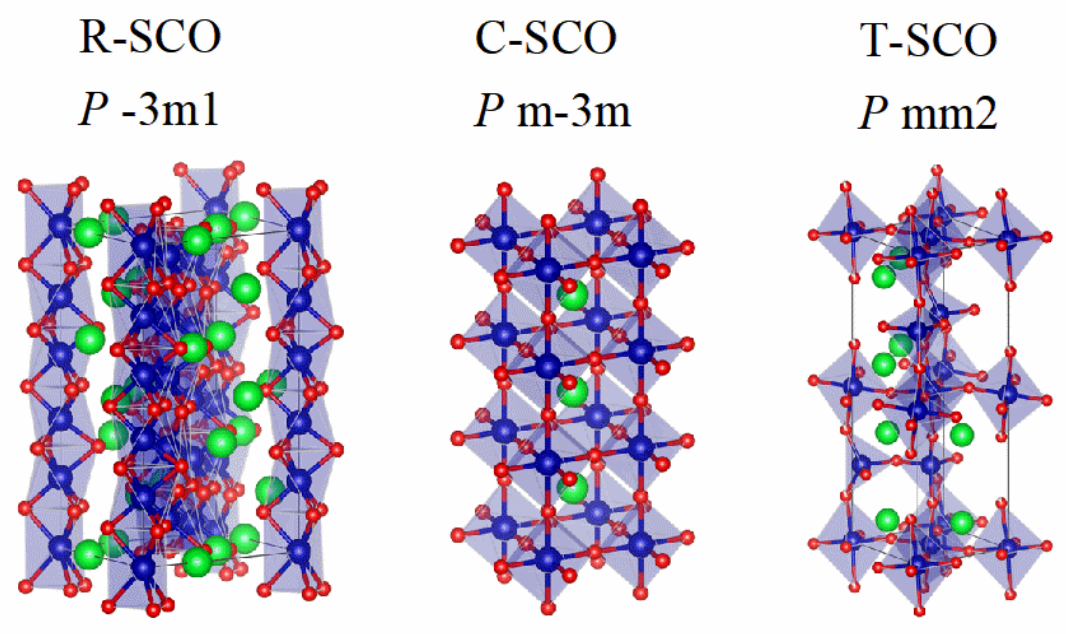

Figure 1. Different crystal structures and space groups of $\mathrm{SrCoO}_{x}$ : face-shared $\mathrm{CoO}_{6}$ in rhombohedral $\mathrm{Sr}_{2} \mathrm{Co}_{2} \mathrm{O}_{5}$ (left), corner-shared $\mathrm{CoO}_{6}$ in cubic $\mathrm{SrCoO}_{3}$ (middle), and corner-shared connections between $\mathrm{CoO}_{6}$ and $\mathrm{CoO}_{4}$ in tetragonal $\mathrm{SrCoO}_{2.5}$ (right). 
In this paper, we report the effect of neodymium $(\mathrm{Nd})$ doping on the magnetic properties of strontium cobaltite due to changes in the crystal structure and oxygen coordination environment. Rietveld analyses provided evidence that varying the $\mathrm{Nd}$ doping in the crystal lattice can induce changes to the topotactic connection style. As a result, the magnetic properties of strontium cobaltite were changed by varying the $\mathrm{Nd}$ concentration. The synchrotron radiation x-ray absorption spectroscopies (XAS) of the Co $L$-edge and $\mathrm{O} K$-edge provide evidence that the electronic structure of the oxygen vacancies in the SCO system are related to change the connections of $\mathrm{CoO}_{6} / \mathrm{CoO}_{4}$ and their magnetic interactions. Therefore, this work proposes a simple method to realize the transformation from face-shared structure to corner-shared octahedron and to improve the ferromagnetic behavior of strontium cobaltite.

\section{Experiment}

The polycrystalline samples of $\mathrm{Sr}_{1-x} \mathrm{Nd}_{x} \mathrm{CoO}_{3}$ (SNCO- $x, x=0,0.05,0.1,0.2,0.3$ ) were prepared using the standard solid-state reaction method. The $\mathrm{SrCO}_{3}, \mathrm{Nd}_{2} \mathrm{O}_{3}$ and $\mathrm{Co}_{2} \mathrm{O}_{3}$ were used as raw materials and weighed according to their molecular formulas. Then, the powders were ball-milled for $24 \mathrm{~h}$, followed by calcination at $900^{\circ} \mathrm{C}$ for 12 h. Finally, the obtained powders were pressed into pellets, followed by sintering at $930^{\circ} \mathrm{C}$ for $12 \mathrm{~h}$. The crystal structures of all the samples were characterized using the Rietveld analyses of the x-ray powder diffraction (XRD) patterns, measured in a Rigaku SmartLab diffractometer with $\mathrm{Cu} \mathrm{K} \alpha$ radiation $(\lambda=1.5406 \AA)$. The temperature and field dependent magnetic moments were determined using a vibrating sample magnetometer (PPMS-VSM, Quantum Design). The electrical structures of Co $L$ edge and $\mathrm{O} K$ edge were characterized by XAS collected at the BL12B-a of the National Synchrotron Radiation Laboratory (NSRL). The total electron yield (TEY) mode using the current measurements from a sample was used. First, the spectrum recorded for the sample was normalized to the photon flux by division through a spectrum obtained for a freshly sputtered gold wafer. Then substrate a line to set the pre-edge to be zero. Finally, the spectra were normalized to yield an edge-jump to one.

\section{Results and discussion}



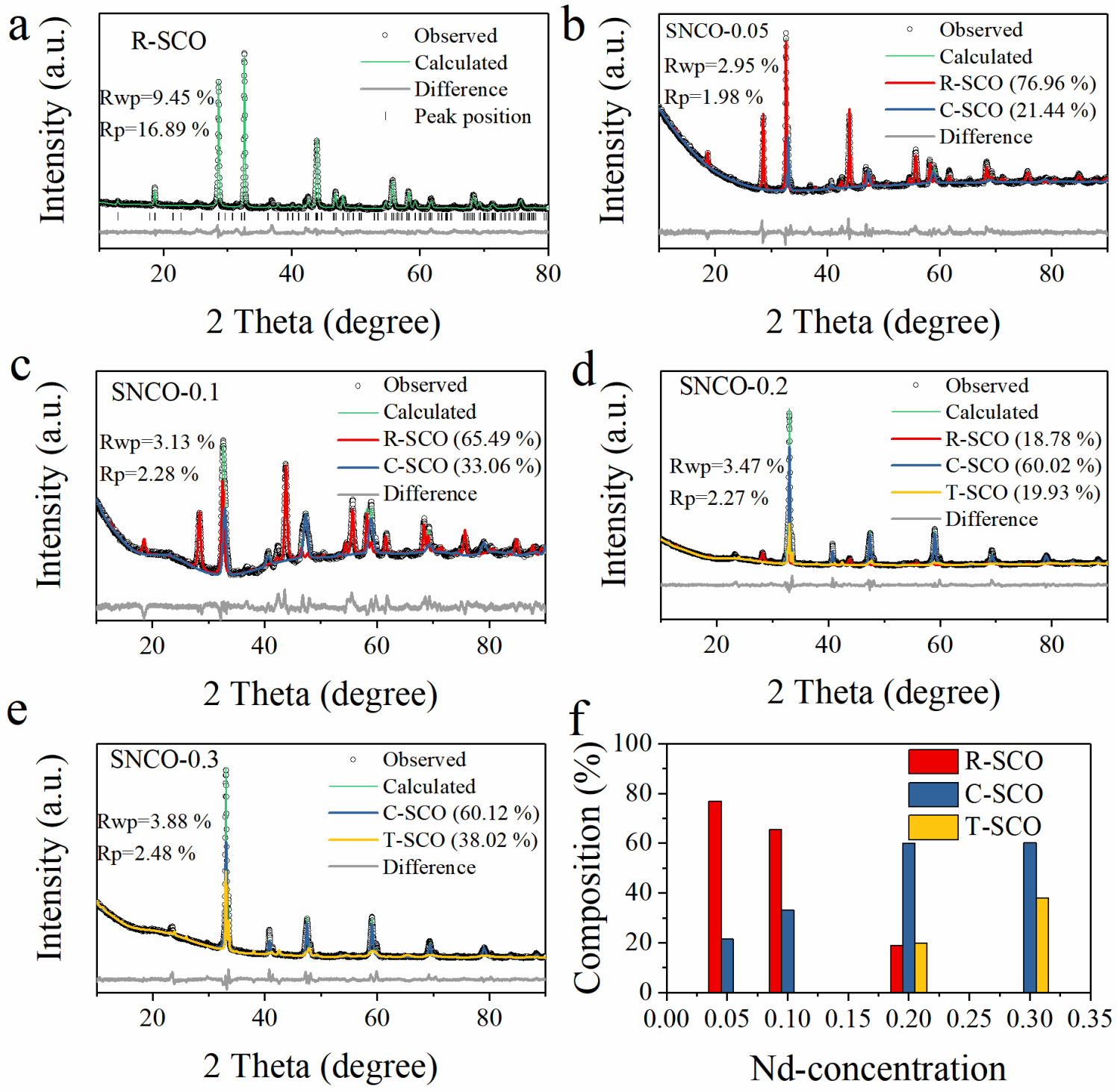

Figure 2. (a-e) The Rietveld analyses of the XRD patterns for samples R-SCO, SNCO-0.05, SNCO0.1, SNCO-0.2, and SNCO-0.3, respectively. (f) Phase composition of SCO with the doping concentration.

Figures 2(a)-(e) show the Rietveld analyses for all the XRD patterns. The results indicate that the crystal structure of the un-doped sample belongs to the rhombohedral system (R-SCO), of which the $\mathrm{CoO}_{6}$ are connected with the face-shared style. The structure of the SCO presented a mixture as different $\mathrm{Nd}$ atoms replaced $\mathrm{Sr}$ in the lattice. For the SNCO-0.05 sample, some of the R-SCO phases transformed into C-SCO (21.44\%), as shown in Fig. 2(b). A small amount of Nd-doping changes the connection style of $\mathrm{CoO}_{6}$ octahedrons. With the increase of $\mathrm{Nd}$-doping concentration to 0.1 , the proportion of R-SCO phase $(65.49 \%)$ decreased and the proportion of C-SCO phase 
(33.06\%) increased, as shown in Fig. 2(c). Figure 2(d) shows the appearance of T-SCO phase $(13.93 \%)$ in sample SNCO-0.2. For sample SNCO-0.3, the R-SCO phase disappeared and the proportion of T-SCO increased, as shown in Fig. 2(e). The refined lattice parameters are summarized in Table 1. With the advent of C-SCO, the R-SCO underwent significant changes, showing that $c$ became significantly larger while $a$ and $b$ became smaller. Figure 2(f) shows how the phase composition of SCO changes with the doping concentration. It can be seen that Nd-doping contributes to corner-shared $\mathrm{CoO}_{6}$. On the one hand, with the increase of $\mathrm{Nd}$-doping concentration, the proportion of face-shared R-SCO decreased, and the proportion of corner-shared C-SCO increased, until the R-SCO phase disappeared completely when the Nd-doping was 0.3. On the other hand, due to the metastability of $\mathrm{Co}^{4+}$, the instable $\mathrm{C}-\mathrm{SCO}$ is prone to have oxygen vacancies and thus converts to T-SCO phase.

Table 1 Refined lattice parameters of all samples

\begin{tabular}{|c|c|c|c|}
\hline \multirow[b]{2}{*}{ Sample } & \multicolumn{3}{|c|}{ Lattice parameter $(\AA)$} \\
\hline & R-SCO & C-SCO & T-SCO \\
\hline \multirow[t]{2}{*}{$x=0$} & $a, b=9.49$ & - & - \\
\hline & $\mathrm{c}=12.39$ & & \\
\hline \multirow[t]{2}{*}{$x=0.05$} & $a, b=9.50$ & 3.86 & - \\
\hline & $\mathrm{c}=12.44$ & & \\
\hline \multirow[t]{2}{*}{$x=0.1$} & $a, b=9.48$ & 3.81 & - \\
\hline & $\mathrm{c}=12.45$ & & \\
\hline \multirow[t]{3}{*}{$x=0.2$} & $a, b=9.41$ & 3.83 & $a=15.20$ \\
\hline & $\mathrm{c}=12.59$ & & $\mathrm{~b}=5.28$ \\
\hline & & & $\mathrm{c}=5.39$ \\
\hline \multirow[t]{3}{*}{$x=0.3$} & - & 3.83 & $a=15.82$ \\
\hline & & & $\mathrm{b}=5.49$ \\
\hline & & & $\mathrm{c}=5.57$ \\
\hline
\end{tabular}


Considering the ionic radii of $\mathrm{Nd}^{3+}, \mathrm{Sr}^{2+}$, and $\mathrm{Co}^{4+} / \mathrm{Co}^{3+} / \mathrm{Co}^{2+}, \mathrm{Nd}$ should replace $\mathrm{Sr}$ which is close to its ionic radius, but not Co which is quite different from its ionic radius. And the ionic radius of $\mathrm{Nd}^{3+}$ is smaller than that $\mathrm{Of}^{2+}$, which may be an important reason for the phase transition of crystal structure from R-SCO to C-SCO. In order to understand the changes of Nd-doping on the electron structure of SCO, Co $L$ edge and $\mathrm{O} K$ edge XAS were characterized. As shown in Figure 3(a), the valence state of Co ions in all samples as a whole is +3 . [13,19] However, all the doped samples have slightly lower energies at the $L_{3}$ - and $L_{2}$-edges when compared to the SCO. This results are contradictory to the valence state of Co ions in SCO. We think that face-shared structures are not prone to oxygen vacancies. The Nd-doping opens the face-shared structure, but there are a large number of oxygen vacancies in the doped samples due to the instability of $\mathrm{Co}^{4+}$. Therefore, the valence state of $\mathrm{Co}$ ions in doped samples is lower than that of un-doped sample $(\mathrm{SCO}, \mathrm{x}=0.0)$. In addition, the position of the peak is independent of the doping concentration, which may also be the result of the decrease in the valence state of Co caused by the presence of oxygen vacancies in C-SCO, and ultimately the effect reflected in the mixture on average.
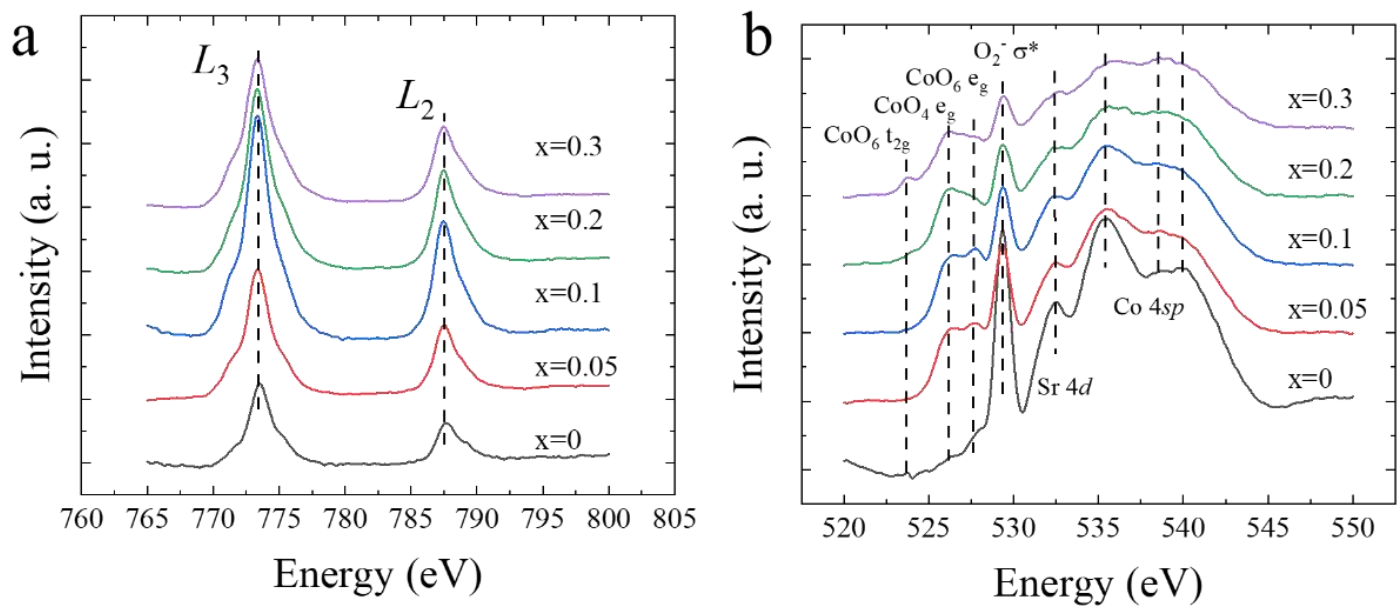

Figure 3. (a) Co $L$-edge XAS for all samples, and (b) O $K$-edge XAS of all samples.

As shown in Fig. 3(b), the $\mathrm{O} K$-edge spectrums have multiple differences, which originate from the excited electrons of the $\mathrm{O} 1 s$ core state to the hole states of the $2 p$ characters. In TMOs, the O $2 p$ state is hybridized with the $s, p$, and $d$ states of its 
neighboring atoms. Figure 3(b) shows all the typical final hybrid states. [19] Among them, the $\mathrm{O}_{2}^{-}$comes from the adsorbed oxygen at the oxygen vacancies. [20,21] Another obvious feature is the relative changes of the $\mathrm{CoO}_{6} t_{2 \mathrm{~g}}$ and $\mathrm{CoO}_{6} e_{\mathrm{g}}$ peaks, which reflect the electrical structure of $\mathrm{Co}$ in the different samples. The $\mathrm{CoO}_{6} t_{2 \mathrm{~g}}$ peak intensity increases, and the $\mathrm{CoO}_{6} e_{\mathrm{g}}$ peak intensity reduces with more $\mathrm{Nd}$ in the lattice. This indicates that some $t_{2 \mathrm{~g}}$ levels in the $\mathrm{CoO}_{6}$ are empty, and some $e_{\mathrm{g}}$ levels are filled with electrons with the increased $\mathrm{Nd}$ doping. Considering that the valence of Co does not change, the partially filled $e_{\mathrm{g}}$ levels may come from the electrons in the $t_{2 \mathrm{~g}}$ levels, which may form intermediate-spin (IS) states. This electronic transition results in the generation of more unpaired electrons, which are responsible for the enhanced effective magnetic moment $\mu_{\text {eff. }}$ The C-SCO is a typical charge-transfer material with the IS ground state, and its ferromagnetic interaction originates from the ferromagnetic $\mathrm{Co}-\mathrm{O}$ Co interaction through an oxygen with an O-2p hole. [22] With the increase of Nddoping concentration in $\mathrm{Sr}_{1-x} \mathrm{Nd}_{x} \mathrm{CoO}_{3}$, more IS states are produced with the face-shared to corner-shared transition. Therefore, the changes of the electronic structure with doping concentration will lead to ferromagnetic enhancement in $\mathrm{Sr}_{1-x} \mathrm{Nd}_{x} \mathrm{CoO}_{3}$.

The zero field cooling (ZFC) and field cooling (FC) curves for all the samples are shown in Fig. 4(a), confirming the enhanced ferromagnetic property. The ZFC and FC curves for the un-doped sample are almost completely coincident at a value that is nearly zero when compared to the other samples. The increased Nd-doping into the lattice shows that the heavily-doped sample has a larger irreversibility between the ZFC and FC curves. All the doped samples have a broad peak below the bifurcation temperature in the ZFC curves, which reflects the glassy-like magnetic behavior in these magnetic systems. [23,24] In general, a broad peak in the ZFC curve is explained by two opposing magnetic effects. On one hand, the magnetization is expected to increase at lower temperatures due to the reduced spin fluctuations. On the other hand, anti-parallel spins tend to reduce the magnetization at lower temperatures. As a result, the two competitive interactions produces a peak. [25] The FC curve shows an increase below the bifurcation temperature, which is similar to ferro- or ferrimagnets. 

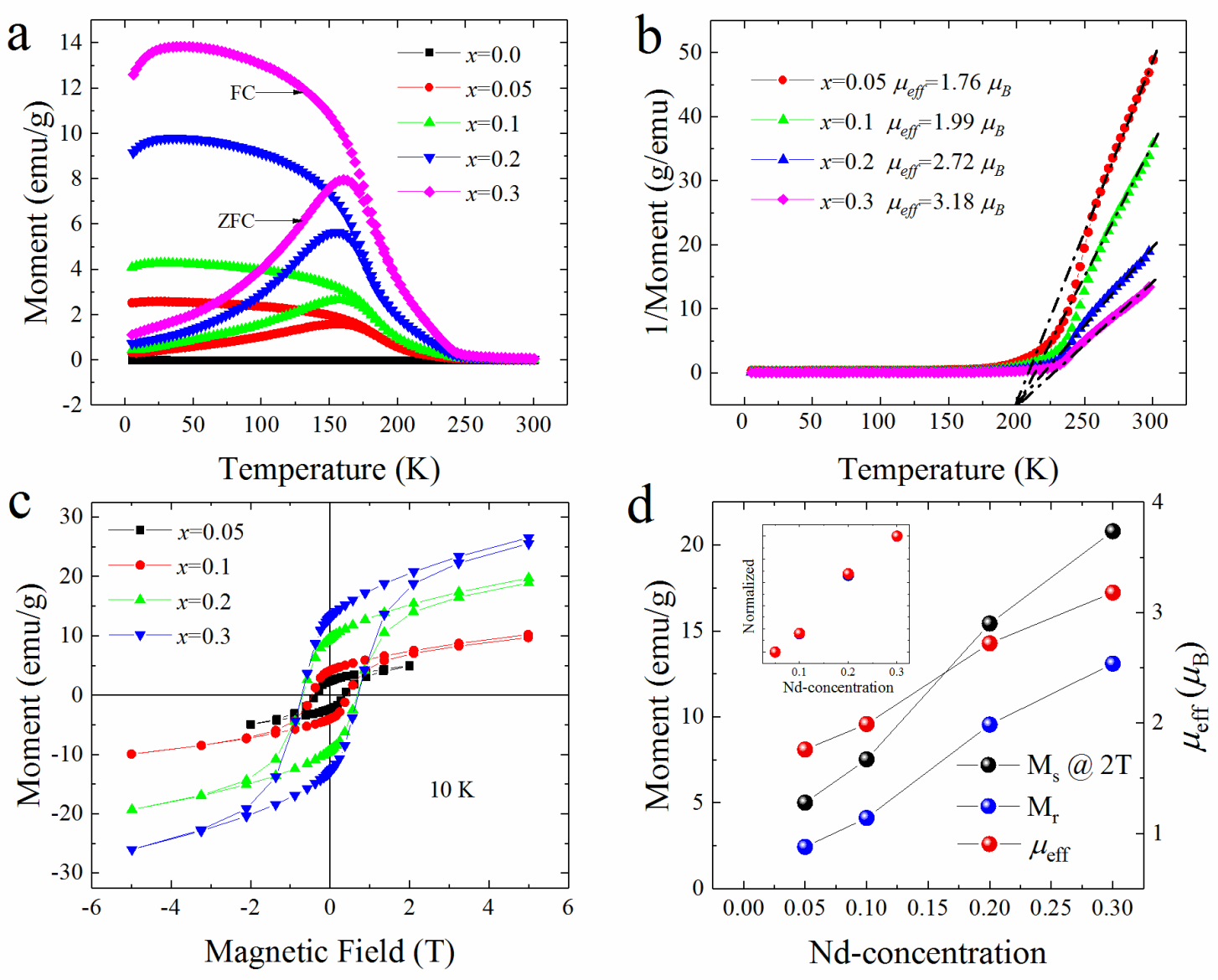

Figure 4. (a) FC and ZFC curves for different Nd concentrations, (b) temperature dependent inverse FC magnetic moment and fitted effective magnetic moment for all doped samples, (c) MH curves at $10 \mathrm{~K}$, (d) effective magnetic moments, the saturation magnetic moment $\left(\mathrm{M}_{\mathrm{s}}\right)$ at $2 \mathrm{~T}$ and residual magnetic moment $\left(\mathrm{M}_{\mathrm{r}}\right)$ from the $\mathrm{MH}$ curves in (c). And the inset of $(\mathrm{d})$ is the normalized values of $\mu_{\mathrm{eff}}, \mathrm{M}_{\mathrm{s}}$, and $\mathrm{M}_{\mathrm{r}}$.

To determine the Curie-Weiss temperature $\left(T_{\mathrm{c}}\right)$ for all the samples, we used the Curie-Weiss law (Eq. 1) to fit the curves using its inverse form (Eq. 2), as shown in Fig. 4(b):

$$
\begin{aligned}
& \chi=\frac{C}{T-T_{C}} \\
& \frac{1}{\chi}=\frac{T-T_{C}}{C}
\end{aligned}
$$

where $\chi, C, T$ and $T_{c}$ represent the magnetic susceptibility, Curie-Weiss constant $(C>$ $0)$, temperature and Curie point, respectively. All the doped samples have the same fitted Curie point of $T_{c} \approx 200 \mathrm{~K}$, as shown in Fig. 4(b), which reflects their identical ferromagnetic source. By fitting the linear portion of the $1 / \chi-T$ curve to obtain the $C$ 
value, the effective magnetic moment can be converted according to the Langevine paramagnetic theory,

$$
\chi=\frac{N g^{2} \mu_{B}^{2}}{3 k T} J(J+1)=\frac{C}{T}
$$

where the effective magnetic moment is $\mu_{\text {eff }}=g \mu_{B} \sqrt{J(J+1)}$. The calculated results are listed in Fig. 4(b), showing that $\mu_{\text {eff }}$ gradually increases with the Nd-doping. This means that the structural changes bring about direct changes in the electronic structure. To quantify the influence of the electronic structure on the magnetic properties of the doped system, we measured the field-dependent magnetic moment $(\mathrm{MH})$ curves of the doped samples at $10 \mathrm{~K}$ for comparison. All the $\mathrm{MH}$ curves show an obvious ferromagnetic-like hysteresis loop, as presented in Fig. 4(c). The shape and size of the hysteresis loop increase as the doping concentration increases, indicating that the $\mathrm{Nd}$ doping can form FM ordering in the system. The saturation magnetic moment $\left(\mathrm{M}_{\mathrm{s}}\right)$ at $2 \mathrm{~T}$ and residual magnetic moment $\left(\mathrm{M}_{\mathrm{r}}\right)$ of the $\mathrm{MH}$ curves are shown in Fig. 4(d). Samples SNCO-0.2 and SNCO-0.3 have similar amounts of C-SCO, but the $\mathrm{M}_{\mathrm{s}}$ and $\mathrm{M}_{\mathrm{r}}$ of sample SNCO-0.3 are larger than those of SNCO-0.2, as show in Fig. 4(c). This suggests that $\mathrm{T}-\mathrm{SCO}$ can introduce ferromagnetic-like interactions into the magnetic system. Previous works have explained this phenomenon with C-SCO FM clusters in the T-SCO AFM matrix, which cannot be detected using XRD. [16] We compared the normalized values of $\mu_{\text {eff }}, M_{\mathrm{s}}$, and $M_{\mathrm{r}}$, and found that they all increased at the same rate, as shown in the inset of Fig. 4(d). Therefore, the ferromagnetism is caused by the increased $\mu_{\text {eff }}$ of Co, which is related to the face-shared to corner-shared transition of $\mathrm{CoO}_{6}$. In general, the $\mu_{\text {eff }}$ is related to the arrangement of the $3 d$ electrons. A larger $\mu_{\text {eff }}$ can be attributed to more unpaired $3 d$ electrons when ignoring the orbital magnetic moment.

\section{Conclusions}

In summary, Nd modified SCO was prepared using a solid state reaction method. The Rietveld analyses of the XRD patterns show the different phase compositions. The MT and MH curves were applied to analyze the magnetic properties in detail. It was 
observed that more Nd doping caused more C-SCO phase appear, which cannot be completely converted to C-SCO because of the appearance of T-SCO. Due to the transformation from the face-shared to corner-shared octahedron, the ferromagnetic properties were enhanced, which may be caused by the $3 d$ electronic structure change.

Acknowledgements This work was supported by the National Science Foundation of China (Grant No. 11704009, 11504358, 51627901), the National Key R\&D Program of China (2016YFA0401004, 2017YFA0402904, 2019YFA0405603), the Open Programs for the Key Science \& Technology Infrastructures of CAS, and the Anhui Initiative in Quantum Information Technologies (AHY100000). The authors thank beamline BL12-a of NSRL for providing the XAS beam times.

\section{References}

[1]. K. Takada, H. Sakurai, E. Takayama-Muromachi, et al. Superconductivity in two-dimensional $\mathrm{CoO}_{2}$ layers, Nature 2003, 422: 53-55.

[2]. J. Wang, J. B. Neaton, H. Zheng, et al. Epitaxial $\mathrm{BiFeO}_{3}$ Multiferroic Thin Film Heterostructures, Science 2003, 299: 1719-1722.

[3]. J. Cong, K. Zhai, Y. Chai, et al. Spin-induced multiferroicity in the binary perovskite manganite $\mathrm{Mn}_{2} \mathrm{O}_{3}$, Nature Communications 2018, 9: 2996.

[4]. C. Wang, H. Seinige, G. Cao, et al. Anisotropic Magnetoresistance in Antiferromagnetic $\mathrm{Sr}_{2} \mathrm{IrO}_{4}$, Physical Review X 2014, 4: 041034.

[5]. T. Yamamoto, K. Shitara, S. Kitagawa, et al. Selective Hydride Occupation in $\mathrm{BaVO}_{3-x} \mathrm{H}_{x}(0.3$ ctive Hydride Occupation in BaVO in BaVO Octahedra, Chem. Mater. 2018, 30: 1566-1574.

[6]. R. Søndenå, S. Stølen, P. Ravindran, et al. Corner- versus face-sharing octahedra in $\mathrm{AMnO}_{3}$ perovskites $(\mathrm{A}=\mathrm{Ca}$, Sr, and Ba), Phys. Rev. B 2007, 75: 184105.

[7]. J. Zhang, D. Meng, H. Huang, et al. Phase competition in the growth of $\mathrm{SrCoO}_{x} / \mathrm{LaAlO}_{3}$ thin films, $A I P$ Advances 2018, 8: 025322.

[8]. J. Pietosa, A. Wisniewski, R. Puzniak, et al. Pressure effect on magnetic and structural properties of $\mathrm{La}_{1-x} \mathrm{Sr}_{x} \mathrm{CoO}_{3-\delta}$, Phys. Rev. B 2009, 79: 214418.

[9]. P. Bezdicka, A. Wattiaux, J.C. Grenier, et al. Preparation and characterization of Fully stoichiometric $\mathrm{SrCoO}_{3}$ by electrochemical oxidation, Zeitschrift Für Anorganische Und Allgemeine Chemie 1993, 619: 7-12.

[10]. M. Abbate, G. Zampieri, J. Okamoto, et al. X-ray absorption of the negative charge-transfer material $\mathrm{SrFe}_{1-x} \mathrm{Co}_{x} \mathrm{O}_{3}$, Phys. Rev. B 2002, 65: 165120.

[11]. T. Takeda, Y. Yamaguchi, H. Watanabe, Magnetic Structure of $\mathrm{SrCoO}_{2.5}$, J. Phys. Soc. Jpn. 1972, 33: $970-$ 972.

[12]. H. Jeen, W. S. Choi, M. D. Biegalski, et al. Reversible redox reactions in an epitaxially stabilized $\mathrm{SrCoO}_{\mathrm{x}}$ oxygen sponge, Nat. Mater. 2013, 12: 1057-1063.

[13]. N. Lu, P. Zhang, Q. Zhang, et al. Electric-field control of tri-state phase transformation with a selective dualion switch, Nature 2017, 546: 124. 
[14]. J. R. Petrie, C. Mitra, H. Jeen, et al. Strain Control of Oxygen Vacancies in Epitaxial Strontium Cobaltite Films, Adv. Funct. Mater. 2016, 26: 1564-1570.

[15]. Y. Long, Y. Kaneko, S. Ishiwata, et al. Synthesis of cubic $\mathrm{SrCoO}_{3}$ single crystal and its anisotropic magnetic and transport properties, J. Phys.: Condens. Matter 2011, 23: 245601.

[16]. A. Muñoz, C. de la Calle, J. A. Alonso, et al. Crystallographic and magnetic structure of $\mathrm{SrCoO}_{2.5}$ brownmillerite: Neutron study coupled with band-structure calculations, Phys. Rev. B 2008, 78: 054404.

[17]. L. Xie, H.L. Huang, Y.L. Lu, Temperature- and magnetic field-dependence of exchange bias in $\mathrm{SrCoO}_{2.29}$ ceramics, AIP Advances 2017, 7: 015207.

[18]. S. Hu, Y. Wang, C. Cazorla, et al. Strain-Enhanced Oxygen Dynamics and Redox Reversibility in Topotactic $\mathrm{SrCoO}_{3-\delta}(0<\delta \leq 0.5)$, Chem. Mater. 2017, 29: 708-717.

[19]. L. Karvonen, M. Valkeapää, R.-S. Liu, et al. O-K and Co-L XANES Study on Oxygen Intercalation in Perovskite $\mathrm{SrCoO}_{3-\delta}$, Chem. Mater. 2010, 22: 70-76.

[20]. N. Yamazoe, Y. Teraoka, T. Seiyama, TPD and XPS study on thermal behavior of absorbed oxygen in $\mathrm{La}_{1-x} \mathrm{Sr}_{x} \mathrm{CoO}_{3}$, Chem. Lett. 1981, 10: 1767-1770.

[21]. Z. Chen, J. Wang, D. Huan, et al. Tailoring the activity via cobalt doping of a two-layer Ruddlesden-Popper phase cathode for intermediate temperature solid oxide fuel cells, Journal of Power Sources 2017, 371: 41-47.

[22]. R.H. Potze, G.A. Sawatzky, M. Abbate, Possibility for an intermediate-spin ground state in the charge-transfer material $\mathrm{SrCoO}_{3}$, Phys. Rev. B 1995, 51: 11501-11506.

[23]. F.C. Chou, N.R. Belk, M.A. Kastner, et al. Spin-Glass Behavior in $\mathrm{La}_{1.96} \mathrm{Sr}_{0.04} \mathrm{CuO}_{4}$, Phys. Rev. Lett. 1995, 75 : 2204-2207.

[24]. T. Park, Z. Nussinov, K.R.A. Hazzard, et al. Novel Dielectric Anomaly in the Hole-Doped $\mathrm{La}_{2} \mathrm{Cu}_{1-x} \mathrm{Li}_{x} \mathrm{O}_{4}$ and $\mathrm{La}_{2}-\mathrm{Sr}_{x} \mathrm{NiO}_{4}$ Insulators: Signature of an Electronic Glassy State, Phys. Rev. Lett. 2005, 94: 017002.

[25]. S.M. Yusuf, M. Sahana, M.S. Hegde, et al. Evidence of ferromagnetic domains in the $\mathrm{La} 0.67 \mathrm{Ca} 0 .{ }_{33} \mathrm{Mn}_{0.9} \mathrm{Fe}_{0.1} \mathrm{O}_{3}$ perovskite, Phys. Rev. B 2000, 62: 1118-1123. 


\section{Figures}

R-SCO

$P-3 \mathrm{~m} 1$

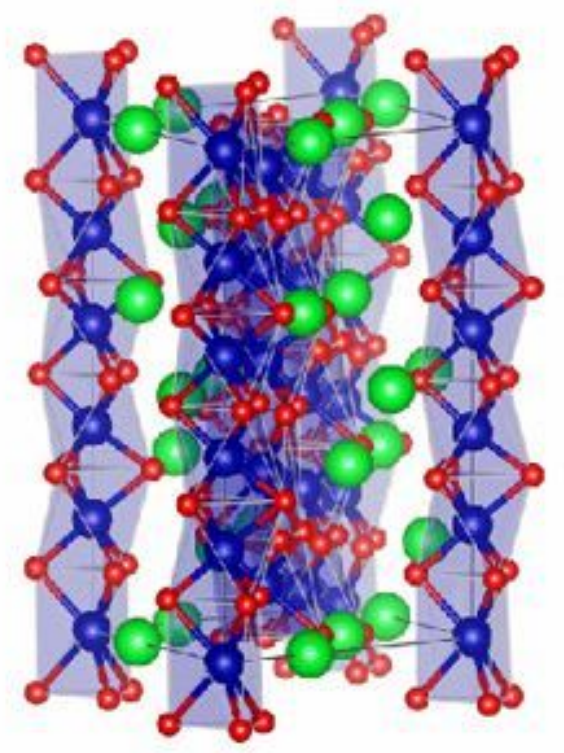

C-SCO

$P \mathrm{~m}-3 \mathrm{~m}$

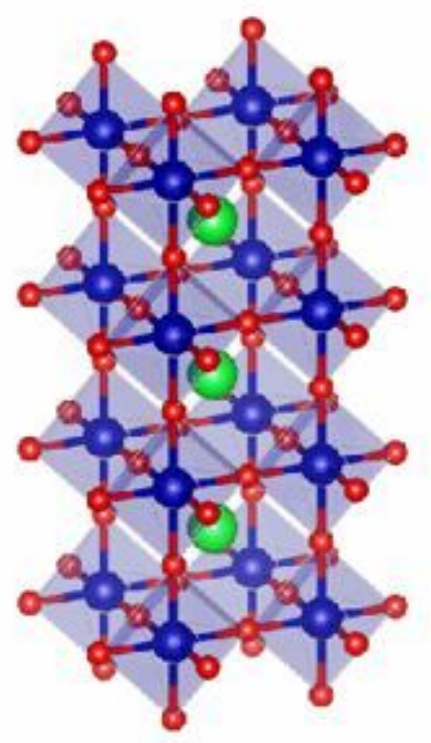

T-SCO

$P \mathrm{~mm} 2$

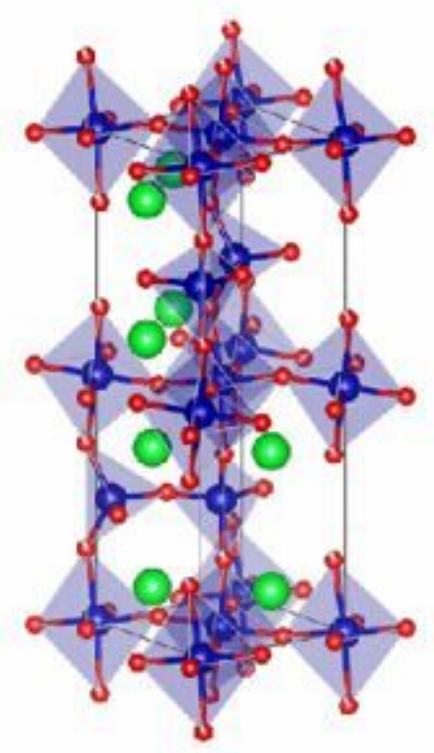

Figure 1

Different crystal structures and space groups of SrCoOx: face-shared $\mathrm{CoO} 6$ in rhombohedral Sr2Co205 (left), corner-shared $\mathrm{CoO} 6$ in cubic $\mathrm{SrCoO} 3$ (middle), and corner-shared connections between $\mathrm{CoO} 6$ and $\mathrm{CoO} 4$ in tetragonal $\mathrm{SrCoO} 2.5$ (right). 



Figure 2

(a-e) The Rietveld analyses of the XRD patterns for samples R-SCO, SNCO-0.05, SNCO-0.1, SNCO-0.2, and SNCO-0.3, respectively. (f) Phase composition of SCO with the doping concentration. 

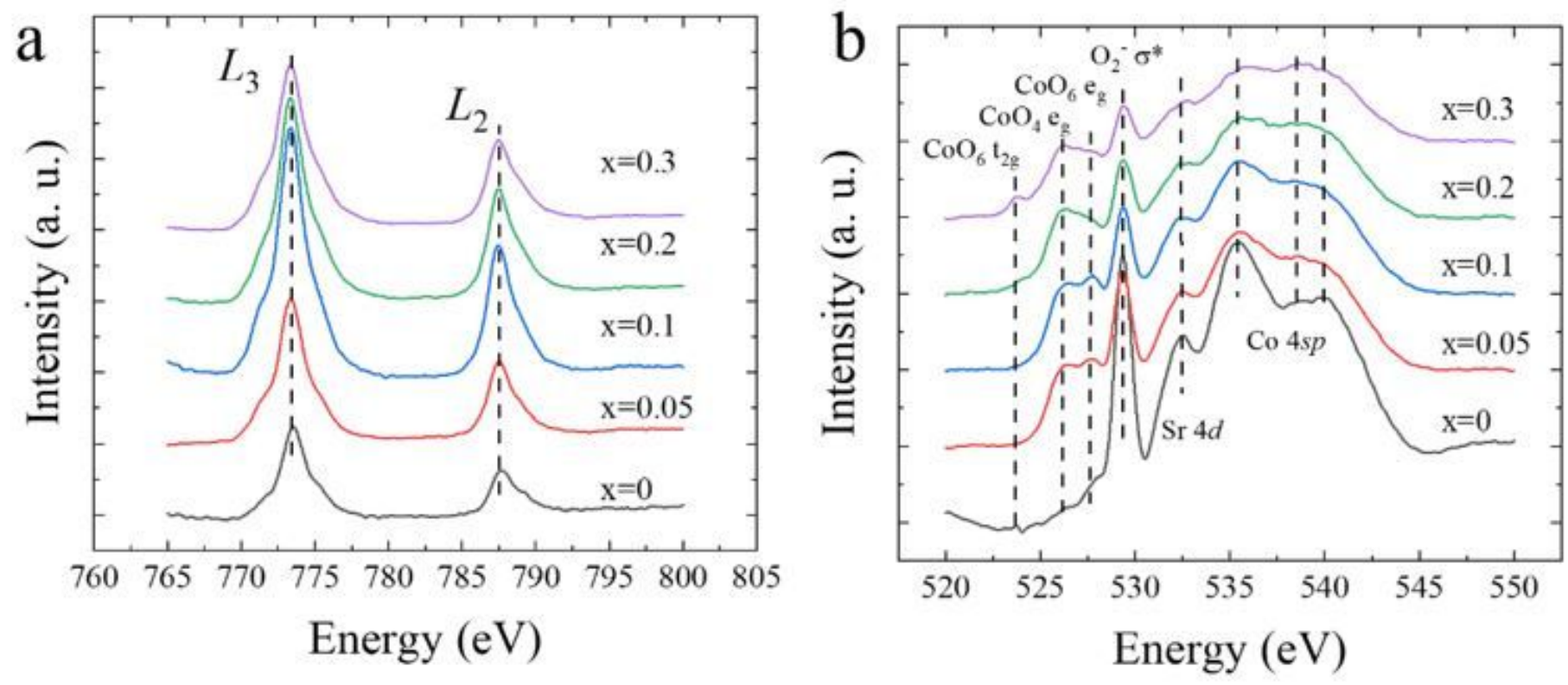

Figure 3

(a) Co L-edge XAS for all samples, and (b) O K-edge XAS of all samples. 

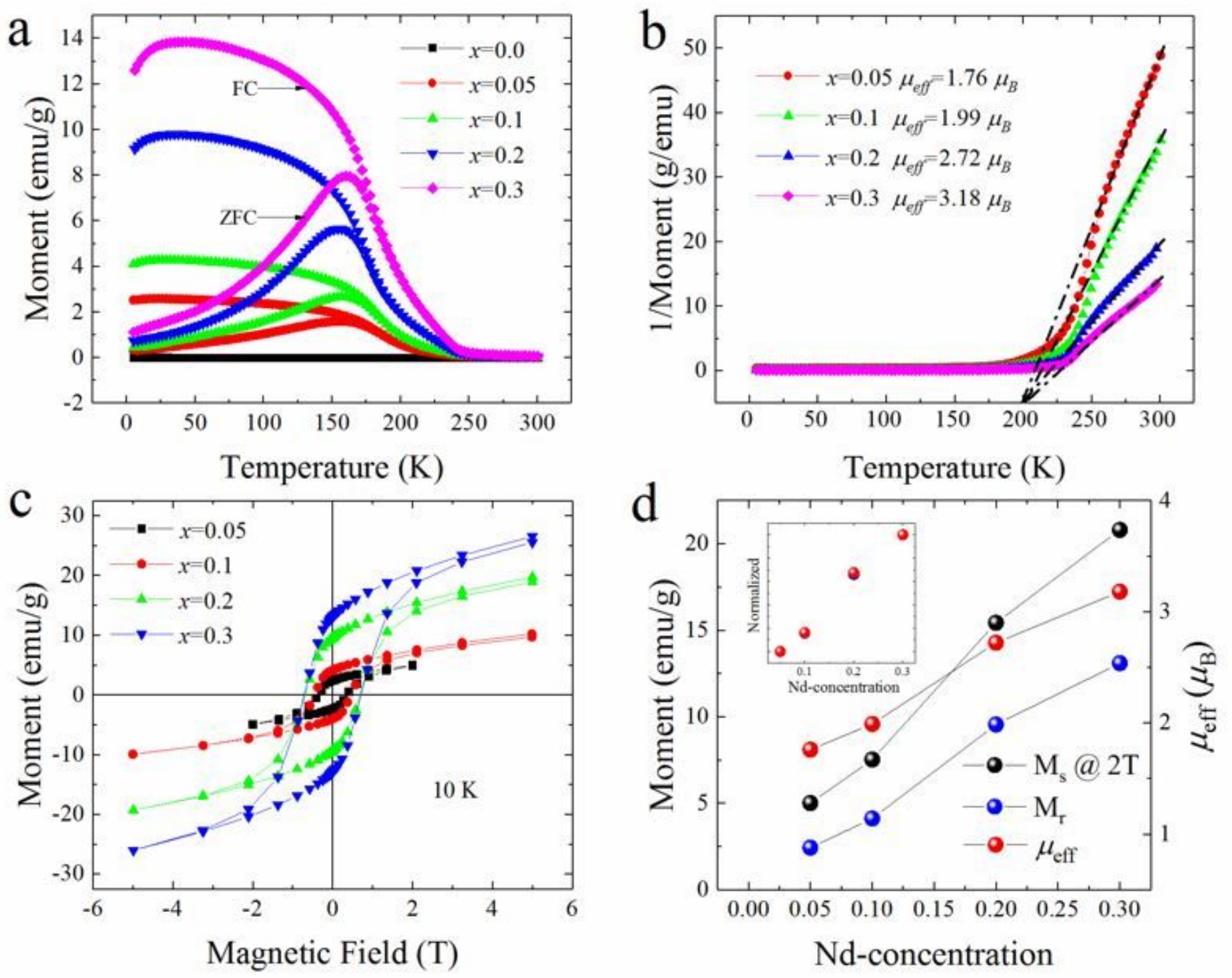

Figure 4

(a) FC and ZFC curves for different Nd concentrations, (b) temperature dependent inverse FC magnetic moment and fitted effective magnetic moment for all doped samples, (c) MH curves at $10 \mathrm{~K}$, (d) effective magnetic moments, the saturation magnetic moment (Ms) at $2 \mathrm{~T}$ and residual magnetic moment (Mr) from the MH curves in (c). And the inset of (d) is the normalized values of $\mu$ eff, Ms, and Mr. 